\title{
Hicksian Surplus Measures of Individual Welfare Change When There is Price And Income UnCertainty
}

\section{by}

Charles Blackorby, David Donaldson, and John A. Weymark

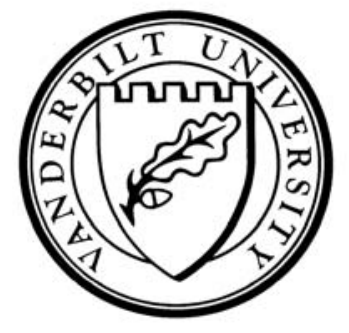

Working Paper No. 06-W18R

August 2006

Revised March 2007

\section{DEPARTMENT OF ECONOMICS \\ VANDERBILT UNIVERSITY \\ NASHVILLE, TN 37235}

www.vanderbilt.edu/econ 


\title{
Hicksian Surplus Measures of Individual Welfare Change When There is Price and Income Uncertainty
}

\author{
Charles Blackorby $^{1}$, David Donaldson ${ }^{2}$, and John A. Weymark ${ }^{3}$ \\ 1 Department of Economics, University of Warwick, Gibbet Hill Road, Coventry \\ CV4 7AL, UNITED KINGDOM and GREQAM, Centre de la Vieille Charité, \\ 2 rue de la Charité, 13002 Marseille, FRANCE. c.blackorby@warwick.ac.uk \\ 2 Department of Economics, University of British Columbia, 997-1873 East Mall, \\ Vancouver, B.C, V6T 1Z1, CANADA. dvdd@telus.net \\ 3 Department of Economics, Vanderbilt University, VU Station B \#35189, 2301 \\ Vanderbilt Place, Nashville, TN 37235-1819, U.S.A. \\ john. weymark@vanderbilt.edu
}

\section{Introduction}

When there is no uncertainty, it is well known that the Hicksian compensating and equivalent variations are exact measures of individual welfare change. That is, the sign of either of these measures of Hicksian consumer's surplus correctly identifies whether a change in prices and income makes an individual consumer better or worse off. ${ }^{4}$ It is also well known that Marshallian consumer's surplus is not an exact measure of individual welfare change except under restrictive assumptions. ${ }^{5}$

The use of the expected value of a Hicksian or Marshallian measure of consumer's surplus to evaluate the welfare consequences of price changes in uncertain environments can be traced back to the seminal analysis of Waugh (1944), who showed that under standard assumptions about individual demand, expected Marshallian consumer's surplus and expected compensating variation are both negative if a stochastic price is stabilized at its arithmetic mean. For an individual whose preferences satisfy the expected utility hypothesis, the use of an expected surplus measure, whether it be Hicksian or Marshallian, is a valid measure of individual welfare change under uncer-

\footnotetext{
${ }^{4}$ Although both the compensating and equivalent variations are exact measures of individual welfare change, they are defined using different reference prices and, hence, do not, in general, assign the same numerical value to a given change in prices and income.

${ }^{5}$ See, for example, Boadway and Bruce (1984, Chapter 7) and Chipman and Moore (1976, 1980).
} 
tainty if and only if its sign correctly determines whether his expected utility increases or decreases as a result of a change in the distribution of prices and incomes across states. Anderson and Riley (1976) have argued that these expected surplus measures do not not correctly track individual preferences when a stochastic price is stabilized unless the marginal utility of income (in the utility representation of preferences used to compute expected utilities) is independent of both the level of income and the value of this price.

Rogerson (1980) and Turnovsky, Shalit, and Schmitz (1980) have identified restrictions on preferences for which expected Marshallian surplus is a valid indicator of individual welfare change when prices and, in the case of Rogerson, incomes are stochastic. For the case in which only one price is uncertain, Helms $(1984,1985)$ has characterized the restrictions on preferences for which expected compensating variation is a valid measure of individual welfare change both when the amount of price variability after the change in the distribution of this price is unrestricted and when the stochastic price is stabilized at its mean value. In each case, these restrictions are quite stringent.

In the models considered by Helms, the consumer allocates a certain income over one or more commodities whose prices are certain and one commodity whose price is uncertain. However, whether uncertainty is generated by, for example, trade shocks (Anderson and Riley, 1976) or by factors that affect the volatility of commodity prices (Newbery and Stiglitz, 1981), it is often the case that the incomes of consumers are also uncertain and one or more prices are state dependent. Furthermore, incomes may be directly affected by random events such as health and the timing of a worker's entry into the labour market, in which case the design of social insurance programmes needs to be evaluated. See, for example, Varian (1980).

In this article, we extend Helms's analyses by identifying the circumstances under which a consumer's surplus criterion based on a Hicksian surplus measure in each state is a valid measure of individual welfare change when income and some or all of the prices vary across states. For concreteness, we use compensating variations in our analysis, but our theorems are also valid for equivalent variations. Although the mechanism that generates a change in the state distribution of prices and incomes can take many forms, for concreteness, we suppose that it is a government project. In order to evaluate the welfare consequences of a project for an individual consumer whose preferences satisfy the expected utility hypothesis, we employ a surplus evaluation function that aggregates the ex post compensating variations in each state into an overall surplus measure. Such a surplus evaluation function is a consistent measure of individual welfare change if it is positive valued whenever the project makes the consumer better off ex ante.

For the kinds of state-dependent prices and incomes that we consider, we show that a consistent measure of individual welfare change based on the ex post compensating variations must regard a project as being welfare improving if and only if its expected compensating variation is positive. Furthermore, the indirect utility function that the consumer uses to evaluate prices and 
income in each state and that is used to compute expected utilities must be affine in income with the origin term a constant and the weight on income independent of those prices that are uncertain. These restrictions imply that preferences are homothetic. If all prices are uncertain, these conditions are inconsistent with the homogeneity properties of an indirect utility function and, hence, we obtain an impossibility result.

In Section 2, we describe our state-contingent alternatives model of uncertainty and formally define the compensating variations obtained in each state. We introduce our consistency criterion and the domains that we consider in Section 3. In Section 4, we adapt a result due to Blackorby and Donaldson (1985) in order to provide a partial characterization of the restrictions implied by consistency. A complete characterization of the restrictions implied by consistency on our domains is established in Section 5 . In Section 6, we discuss our theorems and relate them to results on consistent measures of welfare change that have been obtained in a variety of different contexts. We provide some concluding remarks in Section 7 .

\section{Compensating Variations for the State-Contingent Alternatives Model of Uncertainty}

We employ the state-contingent alternatives model of uncertainty with a finite number of states due to Arrow $(1953,1964)$. For discussions of the expected utility theorem for this model, see Arrow (1965), Blackorby, Davidson, and Donaldson (1977), and Diewert (1993).

We assume that there are $M$ states $(M \geq 2)$ and let $\mathcal{M}=\{1, \ldots, M\}$ denote the set of states. In each state, there are $N$ commodities $(N \geq 2)$. The set of commodities is $\mathcal{N}=\{1, \ldots, N\}$. In state $m$, the prices of the commodities are $p^{m}=\left(p_{1}^{m}, \ldots, p_{N}^{m}\right) \in \mathbb{R}_{++}^{N}$ and the consumer has income $y^{m} \in \mathbb{R}_{+} \cdot{ }^{6}$ Ex ante, the consumer faces the state-contingent price-income vector $(p, y) \in \mathbb{R}_{++}^{M N} \times \mathbb{R}_{+}^{M}$, where $p=\left(p^{1}, \ldots, p^{M}\right)$ and $y=\left(y^{1}, \ldots, y^{M}\right)$.

Ex post consumption in state $m$ is $c^{m}=\left(c_{1}^{m}, \ldots, c_{N}^{m}\right) \in \mathbb{R}_{+}^{N}$. The consumer's ex ante state-contingent consumption vector is $c=\left(c^{1}, \ldots, c^{M}\right) \in$ $\mathbb{R}_{+}^{M N}$. The probability that state $m$ occurs is $\pi_{m}>0$, where $\sum_{m} \pi_{m}=1$. These probabilities can be either subjective or objective, but are fixed throughout our analysis.

We assume that the consumer's preferences over state-contingent commodity vectors in $\mathbb{R}_{+}^{M N}$ are continuous, strictly monotonic, convex, and satisfy the expected utility hypothesis. Hence, these preferences can be represented by a utility function $U: \mathbb{R}_{+}^{M N} \rightarrow \mathbb{R}$ for which

$$
U(c)=\sum_{m} \pi_{m} u\left(c^{m}\right)
$$

\footnotetext{
$\overline{ }^{6} \mathbb{R}_{+}$and $\mathbb{R}_{++}$denote the set of nonnegative and positive numbers, respectively.
} 
for all $c \in \mathbb{R}_{+}^{M N}$, where the function $u: \mathbb{R}_{+}^{N} \rightarrow \mathbb{R}$ is continuous, increasing in each of is arguments, concave, and state independent. Following Arrow (1965), $u$ is called a Bernoulli utility function. ${ }^{7}$ Note that $u$ represents preferences over ex post consumption bundles. Any increasing transform of $u$ also represents these preferences. However, only increasing affine transforms of $u$ are Bernoulli utility functions; i.e., only increasing affine transforms of $u$ can be used to represent the ex ante preferences in the expected utility form given in (1).

The Bernoulli indirect utility function $v: \mathbb{R}_{++}^{N} \times \mathbb{R}_{+} \rightarrow \mathbb{R}$ is defined by setting

$$
v\left(p^{m}, y^{m}\right)=\max _{c^{m} \in \mathbb{R}_{+}^{N}}\left\{u\left(c^{m}\right) \mid p^{m} c^{m} \leq y^{m}\right\}
$$

for all $\left(p^{m}, y^{m}\right) \in \mathbb{R}_{++}^{N} \times \mathbb{R}_{+}$. Hence, the consumer preferences for statecontingent price-income vectors can be represented by the indirect expected utility function $V: \mathbb{R}_{++}^{M N} \times \mathbb{R}_{+}^{M} \rightarrow \mathbb{R}$ defined by setting

$$
V(p, y)=\sum_{m} \pi_{m} v\left(p^{m}, y^{m}\right)
$$

for all $(p, y) \in \mathbb{R}_{++}^{M N} \times \mathbb{R}_{+}^{M}$. It follows from our assumptions on $u$ that the function $v$ is continuous, decreasing, and convex in prices, increasing in income, and homogeneous of degree zero in prices and income. ${ }^{8}$

When there is no price or income uncertainty, with $p=\left(p^{0}, \ldots, p^{0}\right)$ and $y=\left(y^{0}, \ldots, y^{0}\right)$ say, then

$$
V(p, y)=v\left(p^{0}, y^{0}\right)
$$

Thus, $v$ represents preferences over certain price-income vectors. As is the case with $u$, any increasing transform of $v$ represents these preferences over certain outcomes, but only increasing affine transforms of $v$ can be used to compute expected utilities as in (3).

Suppose that the price-income pair in state $m$ is initially $\left(\bar{p}^{m}, \bar{y}^{m}\right)$ and, therefore, the consumer has utility $\bar{u}^{m}=v\left(\bar{p}^{m}, \bar{y}^{m}\right)$ in state $m$. Now consider changing this price-income pair to $\left(\hat{p}^{m}, \hat{y}^{m}\right)$. The consumer then has utility $\hat{u}^{m}=v\left(\hat{p}^{m}, \hat{y}^{m}\right)$ in this state. The compensating variation associated with this change,

$$
s^{m}=S^{m}\left(\bar{p}^{m}, \bar{y}^{m}, \hat{p}^{m}, \hat{y}^{m}\right),
$$

is the maximum amount the consumer would pay for the change. It is defined implicitly by

$$
v\left(\hat{p}^{m}, \hat{y}^{m}-s^{m}\right)=v\left(\bar{p}^{m}, \bar{y}^{m}\right)=\bar{u}^{m} .
$$

${ }^{7}$ A Bernoulli utility function in the state-contingent alternatives model of uncertainty is the analogue of a von Neumann and Morgenstern (1944) utility function in the lottery model of uncertainty.

${ }^{8}$ The function $v$ is decreasing in prices if the value of $v$ decreases when the price of every good is increased. 
Note that

$$
u^{m}=v\left(p^{m}, y^{m}\right) \leftrightarrow y^{m}=e\left(p^{m}, u^{m}\right),
$$

where $e$ is the expenditure function dual to $u$. Thus, the compensating variation in state $m$ can be written as

$$
\begin{aligned}
s^{m} & =e\left(\hat{p}^{m}, \hat{u}^{m}\right)-e\left(\hat{p}^{m}, \bar{u}^{m}\right) \\
& =\hat{y}^{m}-e\left(\hat{p}^{m}, \bar{u}^{m}\right) \\
& =\left[\hat{y}^{m}-\bar{y}^{m}\right]+\left[e\left(\bar{p}^{m}, \bar{u}^{m}\right)-e\left(\hat{p}^{m}, \bar{u}^{m}\right)\right] .
\end{aligned}
$$

Because the expenditure function is increasing in its last argument,

$$
s^{m} \geq 0 \leftrightarrow \hat{u}^{m} \geq \bar{u}^{m} \quad \text { for all } \quad m \in \mathcal{M} .
$$

Therefore, this state-specific measure of willingness-to-pay is nonnegative if and only if the consumer is no worse off in state $m$ as a result of the change from $\left(\bar{p}^{m}, \bar{y}^{m}\right)$ to $\left(\hat{p}^{m}, \hat{y}^{m}\right)$. Hence, the compensating variation correctly identifies whether a change in prices and income in a given state makes the consumer better off or not. This observation is simply a reflection of the well-known fact that the compensating variation is a valid indicator of individual welfare change when there is no uncertainty.

\section{Consistency}

A project affects the consumer by changing the vector of state-contingent prices and incomes. Let $(\bar{p}, \bar{y})$ (resp. $(\hat{p}, \hat{y}))$ denote the pre-project (resp. postproject) prices and incomes. This project changes the consumer's indirect expected utility from $V(\bar{p}, \bar{y})$ to $V(\hat{p}, \hat{y})$. We assume that the same set $D$ of vectors of state-contingent prices and incomes are possible both before and after the implementation of a project.

The question is whether the vector of state-contingent compensating variations $s=\left(s^{1}, \ldots, s^{M}\right)$ defined in (8) can be used to measure the change in the well-being of the consumer for a project that changes $(\bar{p}, \bar{y})$ to $(\hat{p}, \hat{y})$. More precisely, for the domain $D$, we ask if there exists some real-valued function of the state-contingent compensating variations that is positive valued for projects that improve the well-being of the consumer and that is nonpositive for those that do not. This surplus evaluation function is a function $\Gamma: S(D) \rightarrow \mathbb{R}$, where $S(D) \subseteq \mathbb{R}^{M}$ is the set of vectors of state-contingent compensating variations that are achievable when the domain is $D$. We assume that $\Gamma$ is continuous and increasing.

Consistency of the surplus evaluation function with consumer well-being on the domain $D$ is defined as follows.

Consistency. $(\Gamma, V)$ is consistent on $D \subseteq \mathbb{R}_{++}^{M N} \times \mathbb{R}_{+}^{M}$ if and only if

$$
\Gamma\left(s^{1}, \ldots, s^{M}\right) \geq 0 \leftrightarrow V(\hat{p}, \hat{y}) \geq V(\bar{p}, \bar{y})
$$

for all $(\bar{p}, \bar{y}),(\hat{p}, \hat{y}) \in D$. 
Let

$$
D_{y}=\mathbb{R}_{+}^{M}
$$

and, for all $K \subseteq \mathcal{N}$, let

$$
D_{p}^{K}=\left\{p \in \mathbb{R}_{++}^{M N} \mid \forall j \in K, \forall m, m^{\prime} \in \mathcal{M}, p_{j}^{m}=p_{j}^{m^{\prime}}\right\}
$$

and

$$
D^{K}=D_{p}^{K} \times D_{y} .
$$

The sets $D^{K}, K \subseteq \mathcal{N}$, are the domains that we consider for our project evaluations.

For the domain $D^{K}$, the pair $(\Gamma, V)$ has to be consistent for all nonnegative incomes and for all positive prices for which the prices of the goods with indices in the set $K$ are the same in each state. In this domain, the prices with indices in $\mathcal{N} \backslash K$ are permitted to differ across states. Clearly, the more prices that are permitted to differ across states, the more restrictions that $\Gamma$ and $V$ must satisfy.

\section{A Useful Lemma}

By interpreting $\mathcal{M}$ as a set of individuals, instead of a set of states, Blackorby and Donaldson (1985) have defined an indirect Bergson-Samuelson social welfare function $V^{\mathrm{BS}}: \mathbb{R}_{++}^{M N} \times \mathbb{R}_{+}^{M} \rightarrow \mathbb{R}$ by setting

$$
V^{\mathrm{BS}}(p, y)=W\left(v^{1}\left(p^{1}, y^{1}\right), \ldots, v^{M}\left(p^{M}, y^{M}\right)\right)
$$

for all $(p, y) \in \mathbb{R}_{++}^{M N} \times \mathbb{R}_{+}^{M}$, where $W: \mathbb{R}^{M} \rightarrow \mathbb{R}$ is a continuous, increasing Bergson-Samuelson social welfare function and, for all $m \in \mathcal{M}, p^{m}$ are the prices person $m$ faces, $y^{m}$ is his income, and $v^{m}: \mathbb{R}_{++}^{N} \times \mathbb{R}_{+} \rightarrow \mathbb{R}$ is his indirect utility function. Note that individuals may face different prices in (14). As above, we can compute a compensating variation for each individual $m \in \mathcal{M}$ (using the function $v^{m}$ instead of $v$ ) and define consistency (using $V^{\mathrm{BS}}$ instead of $V$ ) as in (10).

Blackorby and Donaldson (1985) have shown that for the domains $D^{\varnothing}$ (all prices can be person specific) and $D^{\mathcal{N}}$ (no price can be person specific), consistency implies that the vector of individual incomes must be separable from the prices in the indirect Bergson-Samuelson social welfare function and that for every vector of compensating variations in the domain of $\Gamma$, the sign of the surplus evaluation function $\Gamma$ must be the same as the sign of a linear function of the individual surplus measures. ${ }^{9}$ Their proofs apply equally well to any domain $D^{K}$ with $K \subseteq \mathcal{N}$.

\footnotetext{
${ }^{9}$ The separability result for the domain $D^{\mathcal{N}}$ was first established by Roberts (1980, Proposition 1).
} 
Because our indirect expected utility function is formally a special case of Blackorby and Donaldson's indirect Bergson-Samuelson social welfare function (with $v^{m}=\pi_{m} v$ and and $V^{\mathrm{BS}}=V$ ), their results also hold for our model. Hence, consistency implies that the state-contingent incomes must be separable from the state-contingent prices in the indirect expected utility function of the consumer and that for every vector of state-contingent compensating variations in $S(D)$, the surplus evaluation function $\Gamma$ must have the same sign as a linear function of these compensating variations.

Lemma 1. For all $K \subseteq \mathcal{N}$, if $(\Gamma, V)$ is consistent on $D^{K}$, then (i) for every $(p, y) \in D^{K}, V$ can be written as

$$
V(p, y)=\bar{V}(p, \phi(y)),
$$

where $\bar{V}$ is continuous, increasing in $\phi(y)$, and homogeneous of degree zero in $(p, y)$ and (ii) there exist $a_{m}>0$ for all $m \in \mathcal{M}$ such that $\phi$ can be written as

$$
\phi(y)=\sum_{m} a_{m} y^{m}
$$

for all $y \in \mathbb{R}_{+}^{N}$. Furthermore,

$$
\Gamma\left(s^{1}, \ldots, s^{M}\right) \geq 0 \leftrightarrow \sum_{m} a_{m} s^{m} \geq 0
$$

for all $\left(s^{1}, \ldots, s^{M}\right) \in S\left(D^{K}\right)$.

For a formal proof of Lemma 1, see Blackorby and Donaldson (1985, Lemma 1, Theorem 1, and Corollary 1.2). The proof strategy is as follows. By considering a project in which only the state-contingent incomes change, (8) implies that the compensating variation in any state is simply the difference between the new and the old income. Because the left side of (10) is independent of prices for such a project, so is the right side, from which the separability result in (15) follows. The homogeneity of degree zero of $V$ implies that $\phi$ can be chosen to be homogeneous of degree one. Using this homogeneity property, it can be shown that $\phi$ satisfies an additive Cauchy equation, whose solution is given by (16). ${ }^{10}$ Because prices have not been changed, the sign of the change in indirect expected utility is the same as the sign of the change in the value of $\phi$, from which (17) follows.

As we have seen, when a project only changes incomes but not prices, the compensating variation in a state is equal to the difference between the preand post-project incomes in that state. Thus, the surplus evaluation function must ignore information about income levels. What Lemma 1 demonstrates is that in order for this function to be sensitive only to income differences and at the same time respect the homogeneity properties of the indirect expected utility function, it must assign each state a weight and then use these weights to compute a weighted sum of compensating variations.

$\overline{10}$ See Aczél (1969, Chapter 2) or Eichhorn (1978, Chapter 1) for an introduction to Cauchy equations. 


\section{The Theorems}

Lemma 1 has identified some restrictions that must be satisfied by the indirect expected utility function $V$ and by the surplus evaluation function $\Gamma$ if they are to be consistent with each other. However, we have yet to identify the restrictions implied by consistency on the Bernoulli indirect utility function $v$ or on the choice of the weights that are used to aggregate the state-contingent compensating variations other than that these weights are positive. In this section, we characterize these restrictions.

The proof of Lemma 1 does not exploit the assumption that $V(p, y)$ is the expected value of the Bernoulli utilities $v\left(p^{m}, y^{m}\right)$ obtained in each state. The conclusions in this lemma are also valid if the ex ante utility $V(p, y)$ is any continuous, increasing function of the ex post utilities $v\left(p^{m}, y^{m}\right)$. We now show that Lemma 1 and the assumption that the consumer's preferences satisfy the expected utility hypothesis imply (i) that the Bernoulli utility function $v$ must be affine in income with the origin term a constant and the weight on income possibly price dependent and (ii) for all vectors of state-contingent compensating variatons in $S(D)$, the sign of the surplus evaluation function must be the same as the sign of the expected value of these compensating variations. The first of these conditions implies that the Bernoulli direct utility function $u$ is homothetic.

Theorem 1. For all $K \in \mathcal{N}$, if $(\Gamma, V)$ is consistent on $D^{K}$, then there exists a function $\alpha: \mathbb{R}_{++}^{N} \rightarrow \mathbb{R}_{++}$and a scalar $\beta$ for which

$$
v\left(p^{0}, y^{0}\right)=\alpha\left(p^{0}\right) y^{0}+\beta
$$

for all $\left(p^{0}, y^{0}\right) \in \mathbb{R}_{++}^{N} \times \mathbb{R}_{+}$, where $\alpha$ is continuous, decreasing, convex, and homogeneous of degree minus one. Furthermore,

$$
\Gamma\left(s^{1}, \ldots, s^{M}\right) \geq 0 \leftrightarrow \sum_{m} \pi_{m} s^{m} \geq 0
$$

for all $\left(s^{1}, \ldots, s^{M}\right) \in S\left(D^{K}\right)$.

Proof. From (3), (15), and (16), we obtain

$$
\bar{V}\left(p, \sum_{m} a_{m} y^{m}\right)=\sum_{m} \pi_{m} v\left(p^{m}, y^{m}\right) .
$$

Consider any $p^{0} \in \mathbb{R}_{++}^{N}$ and let $p^{*}=\left(p^{0}, \ldots, p^{0}\right)$. That is, there is no price uncertainty. Define

$$
\begin{gathered}
z^{m}:=a_{m} y^{m} \quad \text { for all } \quad m \in \mathcal{M}, \\
\hat{v}^{m}\left(z^{m}\right):=\pi_{m} v\left(p^{0}, y^{m}\right),
\end{gathered}
$$


and

$$
\hat{V}\left(\sum_{m} z^{m}\right):=\bar{V}\left(p^{*}, \sum_{m} z^{m}\right) .
$$

Substituting (21), (22), and (23) into (20) yields

$$
\hat{V}\left(\sum_{m} z^{m}\right)=\sum_{m} \hat{v}^{m}\left(z^{m}\right) .
$$

Equation (24) is a Pexider equation whose solution is

$$
\hat{v}^{m}\left(z^{m}\right)=\bar{\alpha}\left(p^{0}\right) z^{m}+\bar{\beta}^{m}\left(p^{0}\right) \text { for all } m \in \mathcal{M},
$$

where $\bar{\alpha}\left(p^{0}\right)>0$ because $\hat{v}^{m}$ is increasing in $z^{m} \cdot{ }^{11}$

Note that

$$
\bar{\beta}^{m}\left(p^{0}\right)=\hat{v}^{m}(0)=\pi_{m} v\left(p^{0}, 0\right) \quad \text { for all } \quad m \in \mathcal{M} .
$$

Define

$$
\beta\left(p^{0}\right):=v\left(p^{0}, 0\right) .
$$

From (26) and (27), we obtain

$$
\bar{\beta}^{m}\left(p^{0}\right)=\pi_{m} \beta\left(p^{0}\right) .
$$

Substituting (25) and (28) into (22) and using (21) yields

$$
\pi_{m} v\left(p^{0}, y^{m}\right)=\bar{\alpha}\left(p^{0}\right) a_{m} y^{m}+\pi_{m} \beta\left(p^{0}\right)
$$

or, equivalently,

$$
v\left(p^{0}, y^{m}\right)=\bar{\alpha}\left(p^{0}\right)\left[\frac{a_{m}}{\pi_{m}}\right] y^{m}+\beta\left(p^{0}\right) .
$$

Because $v$ is state independent and $D_{y}=\mathbb{R}_{+}^{M},(30)$ implies that

$$
a_{m}=\kappa \pi_{m} \quad \text { for all } m \in \mathcal{M},
$$

where $\kappa>0$ because $v$ is increasing in $y^{m}$. Defining

$$
\alpha\left(p^{0}\right):=\kappa \bar{\alpha}\left(p^{0}\right)
$$

yields

$$
v\left(p^{0}, y^{0}\right)=\alpha\left(p^{0}\right) y^{m}+\beta\left(p^{0}\right) .
$$

In order for $v$ to be homogenous of degree zero in prices and income, $\alpha$ must be homogenous of degree minus one and $\beta$ must be homogenous of degree zero.

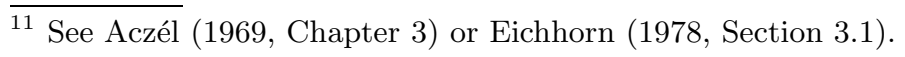


An indirect utility function with the functional form given in (33) is called quasi-homothetic. That is, it exhibits the Gorman (1961) polar form. In order for the demands generated by these preferences to be nonnegative for all prices and incomes, $\beta$ must be independent of prices. See Blackorby, Boyce, and Russell (1978). ${ }^{12}$ Thus, $v$ must satisfy (18). The other properties of $\alpha$ follow straightforwardly from the corresponding properties of $v$.

Using (31), (17) yields (19).

As indicated in the proof of Theorem 1, if $v$ satisfies (30), then it cannot be an indirect utility function unless the function $\alpha$ satisfies the restrictions stated in Theorem 1 and $\beta$ is a constant. However, if we choose a utility level $\bar{u}$ for ex post utility such that the consumption of any good is positive if this utility level is achieved, then it is not necessary for $\beta$ to be a constant if $(\Gamma, V)$ is only required to be consistent on the subset of $D^{K}$ for which ex post utilities are at least $\bar{u}$. Instead, $\beta$ only needs to be continuous, nonincreasing, convex, and homogeneous of degree zero in prices.

Some intuition for Theorem 1 can be obtained by considering the special case in which the Bernoulli indirect utility function $v$ is differentiable. Suppose that there is no price uncertainty and that a project only changes the statecontingent incomes marginally. Let $p^{0}$ be the price vector in each state both before and after the project is implemented, $y$ be the intial state-contingent income vector, and $d y=\left(d y^{1}, \ldots, d y^{M}\right)$ be the vector of income changes that result from this project. Note that $d y$ is also the vector of compensating variations associated with this project. By Lemma 1, consistency requires that

$$
\sum_{m} a_{m} d y^{m} \geq 0 \leftrightarrow \sum_{m} \pi_{m} v_{y}\left(p^{0}, y^{m}\right) d y^{m} \geq 0 .
$$

Because the left side of (34) does not depend on the level of $y$, in order for this equivalence to hold for all $y \in \mathbb{R}_{+}^{M}$ and all $d y$ in a neighbourhood of the origin for which $y+d y \in \mathbb{R}_{+}^{M}$, the marginal utility of income function $v_{y}$ must be positive and cannot depend on income. Hence, $v$ is an increasing affine function of income. That is, $v$ satisfies (33). Using (33), (34) simplifies to

$$
\sum_{m} a_{m} d y^{m} \geq 0 \leftrightarrow \alpha\left(p^{0}\right) \sum_{m} \pi_{m} d y^{m} \geq 0,
$$

which can only hold for all $d y$ in a neighbourhood of the origin if the weights $a=\left(a_{1}, \ldots, a_{M}\right)$ are proportional to the probabilities $\pi=\left(\pi_{1}, \ldots, \pi_{M}\right)$.

A striking feature of Theorem 1 is that consistency requires that projects be evaluated in terms of expected compensating variation. That is, a project

\footnotetext{
${ }^{12}$ This result can be shown quite easily for the case in which $v$ is differentiable. By Euler's Theorem, $\sum_{i=1}^{n} p_{i}^{0} \partial \beta\left(p^{0}\right) / \partial p_{i}^{0}=0$. Hence, if $\beta$ is not independent of prices, there must exist some price vector $\bar{p}^{0}$ and good $j$ for which $\partial \beta\left(\bar{p}^{0}\right) / \partial p_{j}^{0}>0$. Using Roy's Identity, the demand for good $j$ at $\left(\bar{p}^{0}, y^{m}\right)$ is $c_{j}\left(\bar{p}^{0}, y^{m}\right)=-\left[y^{m} \partial \alpha\left(\bar{p}^{0}\right) / \partial p_{j}^{0}+\partial \beta\left(\bar{p}^{0}\right) / \partial p_{j}^{0}\right] / \alpha\left(\bar{p}^{0}\right)$, which is negative when $y^{m}$ is sufficiently close to 0 .
} 
is welfare improving for an individual if and only if the expected compensating variation of the project is positive. As we have noted, previous studies of cost-benefit analysis under uncertainty for a single individual simply assume that the surplus evaluation function is the expected value of some measure of consumer's surplus. For the domains we are considering, we have shown that this must be the case, at least when surplus is measured using the compensating variation. In particular, it is not possible to require the surplus evaluation function to exhibit inequality aversion in the distribution of compensating variations across states.

In Theorem $1, K$ is the set of goods for which prices are certain across states. If $K=\mathcal{N}$, then there is no price uncertainty, whereas if $K=\varnothing$, then all prices can vary across states. Note that any $p \in D_{p}^{K}$ can be written as

$$
p=\left(p_{K}^{0}, p_{-K}^{1}, \ldots, p_{K}^{0}, p_{-K}^{M}\right),
$$

where $p_{K}^{0}$ are the prices of the goods that are certain across states.

Theorem 2 shows that the weight on income in the Bernoulli indirect utility function (18) can only depend on the prices of the goods that are certain. Furthermore, requiring the Bernoulli indirect utility function $v$ to satisfy this restriction and requiring the surplus evaluation function to identify a project as being welfare improving if and only if the expected compensating variation is positive are jointly necessary and sufficient for $(\Gamma, V)$ to be consistent on any of the domains we are considering except for the domain in which all prices are uncertain.

Theorem 2. For all $K \in \mathcal{N} \backslash \varnothing,(\Gamma, V)$ is consistent on $D^{K}$ if and only if (18) and (19) hold and there exists a function $\alpha_{K}: \mathbb{R}_{++}^{|K|} \rightarrow \mathbb{R}_{++}$for which

$$
\alpha\left(p_{K}^{0}, p_{-K}^{0}\right)=\alpha_{K}\left(p_{K}^{0}\right)
$$

for all $\left(p_{K}^{0}, p_{-K}^{0}\right) \in \mathbb{R}_{++}^{N}$, where $\alpha_{K}$ is continuous, decreasing, convex, and homogeneous of degree minus one.

Proof. Suppose that $(\Gamma, V)$ is consistent on $D^{K}$. From (20) and Theorem 1, we have

$$
\bar{V}\left(p, \sum_{m} \pi_{m} y^{m}\right)=\sum_{m} \pi_{m}\left[\alpha\left(p^{m}\right) y^{m}+\beta\right]
$$

for all $(p, y) \in D^{K}$.

Consider any $j \notin K$ and, contrary to the theorem, suppose that there exist distinct $p^{m^{\prime}}, p^{m^{\prime \prime}} \in \mathbb{R}_{++}^{N}$ for which $p_{i}^{m^{\prime}}=p_{i}^{m^{\prime \prime}}$ for all $i \neq j$ and $\alpha\left(p^{m^{\prime}}\right) \neq$ $\alpha\left(p^{m^{\prime \prime}}\right)$. Consider any $\bar{p} \in D_{p}^{K}$ for which $\bar{p}^{m^{\prime}}=p^{m^{\prime}}$ and $\bar{p}^{m^{\prime \prime}}=p^{m^{\prime \prime}}$. Next, consider any distinct $\bar{y}, \hat{y} \in D_{y}$ for which $\bar{y}^{m}=\hat{y}^{m}$ for all $m \neq m^{\prime}, m^{\prime \prime}$ and

$$
\pi_{m^{\prime}} \bar{y}^{m^{\prime}}+\pi_{m^{\prime \prime}} \bar{y}^{m^{\prime \prime}}=\pi_{m^{\prime}} \hat{y}^{m^{\prime}}+\pi_{m^{\prime \prime}} \hat{y}^{m^{\prime \prime}} .
$$


By construction, the value of the left side of (38) is the same when evaluated at $(\bar{p}, \bar{y})$ and $(\bar{p}, \hat{y})$. Thus, $(38)$ implies that

$$
\pi_{m^{\prime}} \alpha\left(p^{m^{\prime}}\right) \bar{y}^{m^{\prime}}+\pi_{m^{\prime \prime}} \alpha\left(p^{m^{\prime \prime}}\right) \bar{y}^{m^{\prime \prime}}=\pi_{m^{\prime}} \alpha\left(p^{m^{\prime}}\right) \hat{y}^{m^{\prime}}+\pi_{m^{\prime \prime}} \alpha\left(p^{m^{\prime \prime}}\right) \hat{y}^{m^{\prime \prime}} .
$$

Because (40) must hold for any nonnegative $\bar{y}^{m^{\prime}}, \bar{y}^{m^{\prime \prime}}, \hat{y}^{m^{\prime}}$, and $\hat{y}^{m^{\prime \prime}}$ that satisfy (39), it follows that $\alpha\left(p^{m^{\prime}}\right)=\alpha\left(p^{m^{\prime \prime}}\right)$, a contradiction. Thus, (37) is satisfied.

The necessity part of the argument is completed by noting that the properties of $\alpha_{K}$ in the theorem statement follow immediately from the properties of $\alpha$ in Theorem 1.

Now, suppose that (18), (19), and (37) are satisfied. Consider any $(\bar{p}, \bar{y})$, $(\hat{p}, \hat{y}) \in D^{K}$, where $(\bar{p}, \bar{y})=\left(\bar{p}_{K}^{0}, \bar{p}_{-K}^{1}, \ldots, \bar{p}_{K}^{0}, \bar{p}_{-K}^{M}, \bar{y}^{1}, \ldots, \bar{y}^{M}\right)$ and $(\hat{p}, \hat{y})=$ $\left(\hat{p}_{K}^{0}, \hat{p}_{-K}^{1}, \ldots, \hat{p}_{K}^{0}, \hat{p}_{-K}^{M}, \hat{y}^{1}, \ldots, \hat{y}^{M}\right)$. Then,

$$
\begin{aligned}
\bar{V}(\hat{p}, \hat{y})-\bar{V}(\bar{p}, \bar{y}) & =\sum_{m} \pi_{m} v\left(\hat{p}_{K}^{0}, \hat{p}_{-K}^{m}, \hat{y}^{m}\right)-\sum_{m} \pi_{m} v\left(\bar{p}_{K}^{0}, \bar{p}_{-K}^{m}, \bar{y}^{m}\right) \\
& =\sum_{m} \pi_{m}\left[\alpha_{K}\left(\hat{p}_{K}^{0}\right) \hat{y}^{m}-\alpha_{K}\left(\bar{p}_{K}^{0}\right) \bar{y}^{m}\right] .
\end{aligned}
$$

Hence,

$$
\bar{V}(\hat{p}, \hat{y})-\bar{V}(\bar{p}, \bar{y}) \geq 0 \leftrightarrow \sum_{m} \pi_{m}\left[\alpha_{K}\left(\hat{p}_{K}^{0}\right) \hat{y}^{m}-\alpha_{K}\left(\bar{p}_{K}^{0}\right) \bar{y}^{m}\right] \geq 0 .
$$

From (6), the compensating variation $s^{m}$ in state $m$ is defined implicitly by

$$
\alpha_{K}\left(\hat{p}_{K}^{0}\right)\left[\hat{y}^{m}-s^{m}\right]+\beta=\alpha_{K}\left(\bar{p}_{K}^{0}\right) \bar{y}^{m}+\beta .
$$

Thus,

$$
s^{m}=\frac{1}{\alpha_{K}\left(\hat{p}_{K}^{0}\right)}\left[\alpha_{K}\left(\hat{p}_{K}^{0}\right) \hat{y}^{m}-\alpha_{K}\left(\bar{p}_{K}^{0}\right) \bar{y}^{m}\right] .
$$

It follows from (42) and (44) that

$$
V(\hat{p}, \hat{y})-V(\bar{p}, \bar{y}) \geq 0 \leftrightarrow \sum_{m} \pi_{m} s_{m} \geq 0,
$$

which completes the sufficiency argument.

In Theorem 2, we have assumed that there is at least one price that is certain. If all prices and incomes can be stochastic, then there is no surplus evaluation function $\Gamma$ that can provide a consistent cost-benefit test on $D^{\varnothing}$ for an individual whose preferences satisfy the expected utility hypothesis.

Theorem 3. There is no function $\Gamma: S\left(D^{\varnothing}\right) \rightarrow \mathbb{R}$ such that $(\Gamma, V)$ is consistent on $D^{\varnothing}$. 
Proof. The necessity part of the proof of Theorem 2 applies equally well when $K=\varnothing$. As a consequence, $\alpha$ must be independent of all prices. That is, for all $\left(p^{0}, y^{0}\right) \in \mathbb{R}_{++}^{N} \times \mathbb{R}_{+}$,

$$
v\left(p^{0}, y^{0}\right)=\xi y^{0}+\beta \quad \text { for some } \quad \xi>0 .
$$

However, if $v$ has this functional form, then it cannot be homogeneous of degree zero and, hence, consistency is impossible.

It is also possible to use Theorem 1 to prove Theorem 3 without relying on Theorem 2. Suppose that $(\Gamma, V)$ is consistent on $D^{\varnothing}$. Project 1 changes the prices and incomes from $(\bar{p}, \bar{y})$ to $(\hat{p}, \hat{y})$. These prices and incomes can be chosen so that the expected compensating variation $\sum_{m} \pi_{m} s^{m}$ of this project is negative and the compensating variation $s^{M}$ in state $M$ is positive for the individual under consideration. Because $(T, V)$ is consistent, Theorem 1 implies that the change in expected utility is negative and, hence, this individual is worse off as a result of Project 1 . Now let $(\tilde{p}, \tilde{y})$ be the vector of prices and incomes for which $\left(\tilde{p}^{m}, \tilde{y}^{m}\right)=\left(\hat{p}^{m}, \hat{y}^{m}\right)$ for all $m \neq M$ and $\left(\tilde{p}^{M}, \tilde{y}^{M}\right)=\lambda\left(\hat{p}^{M}, \hat{y}^{M}\right)$, where $0<\lambda \neq 1$. In Project 2 , prices and incomes are changed from $(\bar{p}, \bar{y})$ to $(\tilde{p}, \tilde{y})$. Because the Bernoulli indirect utility function $v$ is homogenous of degree zero in prices and income, the indirect expected utility is the same with $(\tilde{p}, \tilde{y})$ as it is with $(\hat{p}, \hat{y})$. Therefore, Project 2 makes the individual worse off. Let $\tilde{s}^{M}$ be the compensating variation in state $M$ for this project. Because the expenditure function is homogeneous of degree one in prices, it follows from (8) that $\tilde{s}^{M}=\lambda s^{M}$. The compensating variations for the other states are the same with both projects. Because $\pi_{M}>0$, by choosing $\lambda$ to be sufficiently large, the expected compensating variation for Project 2 is positive, violating consistency.

Because the Bernoulli indirect utility function $v$ is homogeneous of degree zero in prices and income, expected utility is unaffected if the prices and income in each state are divided by the price of good one. With this price normalization, the price of good one is certain and always equal to one. It might seem then that Theorem 3 contradicts the special case of Theorem 2 in which $K=\{1\}$. However, this is not the case. While such a price normalization does not change the expected utility either before or after a project is implemented, it does change the value of the compensating variation in any state for which the post-project price of good one is not initially equal to one. In other words, normalizing by setting the price of good one so that it is always equal to one is innocuous from the perspective of calculating expected utility, but it is not innocuous from the perspective of calculating expected compensated variation. As we have seen, it is for precisely this reason that an impossibility result is obtained when all prices and income can vary across states because we can scale the prices and income in any state without changing the prices 
and income in any other state. This independent scaling is not possible if any price must have the same value in every state. ${ }^{13}$

\section{Discussion}

When the Bernoulli indirect utility function $v$ is differentiable, we can measure the consumer's risk aversion with respect to income with the Arrow (1965)Pratt (1964) coefficient of relative risk aversion:

$$
\rho_{y}\left(p^{0}, y^{0}\right)=-\frac{v_{y y}\left(p^{0}, y^{0}\right)}{v_{y}\left(p^{0}, y^{0}\right)} y^{0}
$$

for all $\left(p^{0}, y^{0}\right) \in \mathbb{R}_{++}^{N} \times \mathbb{R}_{+}$. By Theorem 1 , this coefficient must be identically zero. In other words, the consumer must be risk neutral towards income uncertainty.

Analogous to the Arrow-Pratt coefficient of relative risk aversion $\rho_{y}$ for income, Turnovsky, Shalit, and Schmitz (1980) have defined a coefficient of relative risk aversion $\rho_{p_{i}}$ for the price of good $i$ by taking derivatives with respect to $p_{i}$ instead of with respect to $y$ in (47) and then multiplying the resulting fraction by $p_{i}^{0}$ instead of by $y^{0}$. If it is assumed that the consumer's indirect utility function has the expected utility form given in (3), then the consumer's attitudes towards income and price uncertainty can be measured using the coefficients $\rho_{y}$ and $\rho_{p_{i}}, i \in \mathcal{N}$. These measures are only invariant to increasing affine transforms of the Bernoulli indirect utility function $v$, which are also the class of transforms that do not affect the consumer's ex ante preferences over state-contingent prices and incomes. However, when computing the compensating variation in each state, only the ordinal properties of $v$ are used. As a consequence, if $v^{\prime}$ is any increasing transform of $v$, then the compensating variation associated with a project in any state is the same with $v^{\prime}$ as it is with $v$, even if the risk attitudes associated with $v^{\prime}$ differ from those associated with $v$. Therefore, when the surplus evaluation function in (19) is used to determine whether a project is worthwhile or not, it makes the same recommendations for a consumer whose preferences are characterized by the function $v$ as it does for a consumer whose preferences are characterized by $v^{\prime}$. For this reason, as we have seen, restrictions must be placed on $v$ in order for this cost-benefit test to be consistent. ${ }^{14}$

$\overline{13}$ Also note that the domain obtained from $D^{\varnothing}$ by normalizing the price of good one is not the same as $D^{\{1\}}$. For the domain $D^{\{1\}}$, the price of good one is certain in any price vector $p \in D_{p}^{\{1\}}$, but it need not be the same as the price of good one in some other price vector $q \in D_{p}^{\{1\}}$. However, with the price normalization, not only is the price of good one constant across states in a given state-contingent price vector, it has the same value in every state-contingent price vector.

14 See Helms (1985, p. 609) for similar observations about the use of expected compensating variation as a test for whether stablizing a single stochastic price is beneficial for an individual. 
The restrictions on the Bernoulli indirect utility function $v$ that we have identified for consistency imply that the consumer is risk neutral towards income and that the marginal utility of income does not depend on any price that can vary across states. If there is no price uncertainty and a project only changes incomes, then our cost-benefit test declares a project to be worthwhile if it increases the expected value of income. For a consumer who is risk neutral towards income, this is all that he cares about. However, if the consumer is not risk neutral, then he cares about the distribution of incomes, not just its expected value, and consistency would be lost. If a project also changes prices, by using the compensating variation to measure the surplus in each state, price changes are converted into an equivalent income change using the post-project prices. In order for expected compensating variation to provide a consistent cost-benefit test when some of the prices are stochastic, the marginal utility of income must be constant across states. ${ }^{15}$ Because any distribution of incomes across states is possible, this requires that the marginal utility of income be independent of any price that can be state dependent.

In a model with a continuum of states, Helms (1984) has investigated when expected compensating variation is a consistent measure of individual welfare change when the only source of uncertainty is in the price of one good and there are no restrictions on the stochastic variability that this price might exhibit. Helms has shown that risk neutrality towards income and independence of the marginal utility of income with respect to this price are necessary and sufficient for consistency provided that the demand for this good is positive. That is, the Bernoulli indirect utility function must satisfy (33) with $\alpha$ independent of the price that is stochastic. ${ }^{16}$

Our theorems are closely related to results about the consistency of costbenefit tests based on compensating or equivalent variations established by Blackorby, Donaldson, and Moloney (1984) and Blackorby and Donaldson $(1985,1986)$ in a variety of contexts.

Blackorby and Donaldson (1985) have shown (i) that no continuous, increasing surplus evaluation function defined on individual compensating or equivalent variations can be consistent with an indirect Bergson-Samuelson social welfare function when all prices and incomes can be person specific and (ii) that when everyone faces the same prices, consistency requires individual preferences to be quasi-homothetic with everyone having the same price-dependent weight on income. ${ }^{17}$ The latter condition is the necessary and

$\overline{15}$ In $(44), \alpha_{K}\left(\hat{p}_{K}^{0}\right)$ is the marginal utility of income at the post-project prices. If this value depends on any price that is not certain, then it could not be factored out in going from (44) to (45).

${ }^{16}$ Neither Helms (1984) nor the other articles considered in the rest of this section take account of the restrictions required to ensure that demands are nonnegative for all admissible prices and incomes. For this reason, they only show that preferences must be quasi-homothetic, rather than being fully homothetic.

17 Related results may be found in Hammond (1977, 1980) and Roberts (1980). 
sufficient condition identified by Gorman (1953) for the existence of community indifference curves. ${ }^{18}$

In Blackorby, Donaldson, and Moloney (1984), a single consumer, whose utility is a continuous, increasing function of the instantaneous utilities obtained from his consumption in each of a finite number of periods, chooses these consumptions to maximize lifetime utility given the prices of the goods and his wealth in a perfect capital market. They have shown (i) that no discounted sum of the compensating or equivalent variations in each period can serve as a consistent measure of welfare change for such a consumer if prices are free to vary across periods and (ii) that the instantaneous preferences must be quasi-homothetic if all prices are constant across periods. ${ }^{19}$

In Blackorby and Donaldson (1986), there is a single period and each individual consumes an amount of a single commodity should he live, which occurs with positive probability. For the case in which each person's preferences satisfy the expected utility hypothesis and everyone has some level of consumption that makes life just worth living, they have shown (i) that the sum of the individual compensating or equivalent variations is not consistent with the ranking of alternative distributions of survival probabilities and of consumptions obtained with any continuous, increasing Bergson-Samuelson social welfare function if both the probabilities of survival and the consumptions can be person specific and (ii) that when everyone has the same survival probability, then consistency requires that the individual preferences to be quasi-homothetic with utility functions that are affine in consumption with an income weight that can only depend on the common survival probability. As Blackorby and Donaldson (1986, Section III) have noted, the probabilities in this model correspond to prices in the riskless multi-good model.

There is clearly a close family resemblance between these results and those obtained here. This is not surprising. Although our model and those described above differ in some important respects, the overall measure of individual or social welfare in each case is a continuous, increasing function of the utility functions that are used to compute the individual or state-contingent or period-contingent compensating or equivalent varitions. Furthermore, the surplus evaluation function is, in each case, a continuous, increasing function of these surpluses. It is these common structural features of these models that accounts for the similarity of the results about the consistency of welfare evaluations based on Hicksian measures of consumer's surplus that are obtained with them.

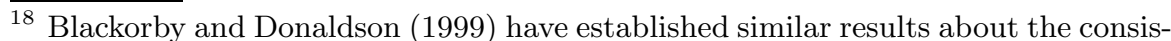
tency of the sum of individual Marshallian consumers' surpluses with an indirect Bergson-Samuelson indirect social welfare function. For discussions of the use of expected Marshallian consumer's surplus as a measure of individual welfare change, see Rogerson (1980), Turnovsky, Shalit, and Schmitz (1980), and Stennek (1999).

${ }^{19}$ The analogue of a perfect capital market in our model is a perfect insurance market that permits an individual to transfer wealth across states. 


\section{Concluding Remarks}

The restrictions on preferences that Helms $(1984,1985)$ has shown are required for expected compensated variation to be a consistent measure of individual welfare change are much less restrictive when a single stochastic price is stabilized at its mean value compared with the case in which all distributions can be stochastic. However, they are still quite stringent and are unlikely to be satisfied in practice. For the prices that are allowed to vary across states and for income, we have placed no restrictions on the pre- and post-project distributions. We could instead restrict our domains by, for example, considering projects that stabilize income or some of the prices. On such domains, the conditions required for consistency would be weaker than those obtained here. However, Helms's theorems suggest that they will nevertheless be quite restrictive, so considering more specialized domains does not appear to be a promising direction in which to seek more positive results.

In view of the rather stringent conditions required for a surplus evaluation function based on the ex post compensating variations to be a consistent measure of individual welfare change, it is natural to ask if there is any measure of consumer's surplus that applies more generally when prices or incomes are uncertain. An affirmative answer is provided by the ex ante compensating and equivalent variations introduced by Schmalensee (1972). ${ }^{20}$

The ex ante compensating variation $s_{c}$ for a project that changes the statecontingent prices and income from $(\bar{p}, \bar{y})$ to $(\hat{p}, \hat{y})$ is defined implicitly by

$$
\sum_{m} \pi_{m} v\left(\hat{p}^{m}, \hat{y}^{m}-s_{c}\right)=V(\bar{p}, \bar{y}) .
$$

That is, $s_{c}$ is the amount by which an individual's income can be reduced in each state in order for the post-project situation to give him the same ex ante expected utility as is achieved before the project is implemented. Because $v$ is increasing in income, $s_{c}$ is positive if and only if the project makes the consumer better off ex ante. Thus, $s_{c}$ can serve as an exact measure of welfare change for any individual whose preferences satisfy the expected utility hypothesis. Similarly, the ex ante equivalent variation $s_{e}$ is the amount of income that needs to be provided to an individual in each state in the pre-project situation in order to give him the same ex ante expected utility as is achieved after the project is implemented. It too is an exact measure of individual welfare change.

Schmalensee (1972) did not advocate the use of these ex ante measures because he thought that they are non-operational. However, assuming that the appropriate coefficients of risk aversion can be determined from analyzing

\footnotetext{
${ }^{20}$ Schmalensee refers to these measures as compensating and equivalent option prices. An alternative proposal for measuring individual welfare change under uncertainty may be found in Boadway and Bruce (1984, Chapter 7).
} 
behaviour under uncertainty, Anderson (1979) has argued that these measures are operational, and so has endorsed their use, as has Helms (1985). ${ }^{21}$ Given that information about risk attitudes is needed in order to determine if expected compensating (or equivalent) variation is a consistent measure of individual welfare change, it therefore seems that there is little reason to use the expected value of some consumer's surplus measure to evaluate projects that involve price and income uncertainty instead of the ex ante compensating or equivalent variation.

\section{Acknowlegements}

This article was presented at the International Conference on Rational Choice, Individual Rights and Non-Welfaristic Normative Economics in Honour of Kotaro Suzumura at Hitotsubashi University, the Workshop on Advances in Collective Choice at the University of Maastricht, the Workshop on Economic Decisions at the Public University of the Navarre, the Workshop on Advances in Microeconomics and Social Choice Theory at the University of Verona, the Eighth International Meeting of the Society for Social Choice and Welfare in Istanbul, and at a seminar at GREQAM. We are grateful for the comments that we have received on these occasions. We are particularly grateful to Peter Hammond for his comments at the Hitotsubashi conference.

\section{References}

Aczél, J. (1969). Lectures on Functional Equations and Their Applications. Academic Press, New York.

Anderson, J. E. (1979). On the measurement of welfare cost under uncertainty. Southern Economic Journal, 45, 1160-1171.

Anderson, J. E. and Riley, J. G. (1976). International trade with fluctuating prices. International Economic Review, 17, 76-97.

Arrow, K. J. (1953). Le rôle des valeurs boursières pour la répartition la meillure des risques. In Econométrie, pages 41-47. Centre National de la Recherche Scientifique, Paris. Translated as Arrow (1964).

Arrow, K. J. (1964). The role of securities in the optimal allocation of risk-bearing. Review of Economic Studies, 31, 91-96.

Arrow, K. J. (1965). Aspects of the Theory of Risk-Bearing. Yrjö Jahnssonin säätiö, Helsinki.

Blackorby, C. and Donaldson, D. (1985). Consumers' surpluses and consistent costbenefit tests. Social Choice and Welfare, 1, 251-262.

${ }^{21}$ The relationship between these ex ante measures and the expected value of a Hicksian or Marshallian measure of ex post consumer's surplus has been explored in Choi and Johnson (1987) and Stennek (1999). 
Blackorby, C. and Donaldson, D. (1986). Can risk-benefit analysis provide consistent policy evaluations of projects involving loss of life? Economic Journal, 96, 758773.

Blackorby, C. and Donaldson, D. (1999). Market demand curves and DupuitMarshall consumers' surpluses: A general equilibrium analysis. Mathematical Social Sciences, 37, 139-163.

Blackorby, C., Davidson, R., and Donaldson, D. (1977). A homiletic exposition of the expected utility hypothesis. Economica, 44, 351-358.

Blackorby, C., Boyce, R., and Russell, R. R. (1978). Estimation of demand systems generated by the Gorman polar form: A generalization of the S-branch utility tree. Econometrica, 46, 345-363.

Blackorby, C., Donaldson, D., and Moloney, D. (1984). Consumer's surplus and welfare change in a simple dynamic model. Review of Economic Studies, 51, 171-176.

Boadway, R. W. and Bruce, N. (1984). Welfare Economics. Basil Blackwell, Oxford.

Chipman, J. S. and Moore, J. C. (1976). The scope of consumer's surplus arguments. In A. M. Tang, F. M. Westfield, and J. S. Worley, editors, Evolution, Welfare, and Time in Economics: Essays in Honor of Nicholas Georgescu-Roegen, pages 69-123. Lexington Books, Lexington, MA.

Chipman, J. S. and Moore, J. C. (1980). Compensating variation, consumer's surplus, and welfare. American Economic Review, 70, 933-949.

Choi, E. K. and Johnson, S. R. (1987). Consumer's surplus and price uncertainty. International Economic Review, 28, 407-411.

Diewert, W. E. (1993). Symmetric means and choice under uncertainty. In W. E. Diewert and A. O. Nakamura, editors, Essays in Index Number Theory, volume 1, pages 355-433. North-Holland, Amsterdam.

Eichhorn, W. (1978). Functional Equations in Economics. Addison-Wesley, Reading, MA.

Gorman, W. M. (1953). Community preference fields. Econometrica, 21, 63-80.

Gorman, W. M. (1961). On a class of preference fields. Metroeconomica, 13, 53-56.

Hammond, P. J. (1977). Dual interpersonal comparisons of utility and the welfare economics of income distribution. Journal of Public Economics, 7, 51-71.

Hammond, P. J. (1980). Dual interpersonal comparisons of utility and the welfare economics of income distribution: A corrigendum. Journal of Public Economics, 14, 105-106.

Helms, L. J. (1984). Comparing stochastic price regimes: The limitations of expected surplus measures. Economics Letters, 14, 173-178.

Helms, L. J. (1985). Expected consumer's surplus and the welfare effects of price stabilization. International Economic Review, 26, 603-617.

Newbery, D. M. and Stiglitz, J. E. (1981). The Theory of Commodity Price Stabilization: A Study in the Economics of Risk. Clarendon Press, Oxford.

Pratt, J. W. (1964). Risk aversion in the small and in the large. Econometrica, 32, $122-136$.

Roberts, K. (1980). Price-independent welfare prescriptions. Journal of Public Economics, 13, 277-297.

Rogerson, W. P. (1980). Aggregate expected consumer surplus as a welfare index with an application to price stabilization. Econometrica, 48, 423-436.

Schmalensee, R. (1972). Option demand and consumer's surplus: Valuing price changes under uncertainty. American Economic Review, 62, 813-824. 
Stennek, J. (1999). The expected consumer's surplus as a welfare measure. Journal of Public Economics, 73, 265-288.

Turnovsky, S. J., Shalit, H., and Schmitz, A. (1980). Consumer's surplus, price instability, and consumer welfare. Econometrica, 48, 135-152.

Varian, H. R. (1980). Redistributive taxation as social insurance. Journal of Public Economics, 14, 49-68.

von Neumann, J. and Morgenstern, O. (1944). Theory of Games and Economic Behavior. Princeton University Press, Princeton.

Waugh, F. V. (1944). Does the consumer benefit from price instability? Quarterly Journal of Economics, 58, 602-614. 